Bundesgesundheitsbl 2019 • 62:1032-1037 https://doi.org/10.1007/s00103-019-02893-2 Online publiziert: 23. April 2019

(c) Springer-Verlag GmbH Deutschland, ein Teil von Springer Nature 2019

Empfehlung des Umweltbundesamtes

\title{
Systemische Untersuchungen von Trinkwasser-Installationen auf Legionellen nach Trinkwasser- verordnung - Probennahme, Untersuchungsgang und Angabe des Ergebnisses
}

\section{Empfehlung des Umweltbundesamtes nach Anhörung der Trinkwasserkommission}

Die Probennahme ist gemäß DIN EN ISO 19458 [1], Tabelle 1, Zweck b) durchzuführen. Hierzu sind nach TrinkwV $₫ 14$ b Absatz 3 geeignete Probennahmearmaturen an repräsentativen und für die Probennahme geeigneten Probennahmestellen durch den Betreiber vorzuhalten (weitere Details hierzu siehe Pkt. 4 und 5).

Nach $\$ 15$ Absatz la TrinkwV ist für die mikrobiologische Bestimmung von Legionellen, spätestens ab dem 1. März 2019, das Verfahren nach ISO 11731 [2, 3] anzuwenden $^{3}$. Die Empfehlung des Umweltbundesamtes aus dem Jahr $2012^{4}$ ist nicht mehr aktuell und musste den neuen Anforderungen angepasst werden. Diese neue Empfehlung dient der Festlegung und Beschreibung des Vorgehens bei der Probennahme und des Untersuchungsganges im Laborbereich und damit der Klarstellung zur Umsetzung der

\footnotetext{
3 Nach der Veröffentlichung der TrinkwV ist die inhaltsgleiche deutsche Übersetzung DIN EN ISO11731:2018-03 erschienen, die jedoch aktuell berichtigt wird. Da sich die TrinkwV auf die ISO-Norm bezieht, wird im Folgenden auch nur auf die ISO11731 verwiesen.

${ }^{4}$ Empfehlung des Umweltbundesamtes nach Anhörung der Trinkwasserkommission vom 23.August 2012: Systemische Untersuchungen von Trinkwasser-Installationen auf Legionellen nach Trinkwasserverordnung. Probennahme, Untersuchungsgang und Angabe des Ergebnisses.
}

Vorgaben der Trinkwasserverordnung in Verbindung mit den allgemein anerkannten Regeln der Technik (a.a.R.d. T). Die Empfehlung des Umweltbundesamtes „Systemische Untersuchungen von Trinkwasser-Installationen auf Legionellen nach Trinkwasserverordnung" vom 23.08.2012 wird hiermit zurückgezogen.

Diese Empfehlung richtet sich an alle, die in die Untersuchung von Trinkwasser-Installationen in Gebäuden auf Legionellen einbezogen sind, insbesondere an die UsI derartiger Anlagen, an Trinkwasseruntersuchungsstellen und Probennehmer sowie an die Mitarbeiterinnen und Mitarbeiter von Gesundheitsämtern.

\section{Begriffsbestimmung}

\subsection{Systemische Untersuchung}

Die systemische Untersuchung gemäß $\$ 14$ b TrinkwV entspricht einer orientie-

Der Textentwurf wurde von Dipl.-Chem. U. Rädel, Dr. C. Förster, Prof. Dr. M. Exner, Dipl.-Ing. F. Hahn, Dipl.-Biol. A. Hummel, Dr. D. Petersohn, Dr.-Ing. B. Wricke und den hinzugezogenen Sachverständigen Dr. J. Fleischer, Dr. B. Frei, Dipl.-Ing. M. Funcke, Dr. S. Huber, Dr. C. Koch, Dipl.-Biol. B. Lange, Dr. K. Luden, Dr. S. Pleischl erarbeitet. Diese Empfehlung wurde von der Trinkwasserkommission am 18.12.2018 verabschiedet. 
renden Untersuchung, wie sie im DVGWArbeitsblatt W 551 [4] beschrieben wird. Der Begriff „systemisch“verdeutlicht, dass es nicht um die Feststellung der Legionellenfreiheit an allen lokalen Entnahmestellen geht, sondern um die Überwachung der Trinkwasser-Installation in der Gesamtheit. Das Ziel ist eine mögliche Kontamination mit Legionellen in Teilen der Trinkwasser-Installation festzustellen, die einen Einfluss auf eine größere Anzahl an Entnahmestellen haben kann, insbesondere in den zentralen Teilen der Trinkwasser-Installation, wie Trinkwassererwärmern, Verteilern, Steigsträngen oder Zirkulationsleitungen.

\subsection{Lokale Kontamination}

Eine lokale Kontamination bezieht sich auf eine Verkeimung einer einzelnen Entnahmearmatur mit Legionellen (z. B. eines Duschkopfes oder eines Duschschlauchs). Der Einfluss einer lokalen Kontamination auf benachbarte Entnahmearmaturen oder Teile der Trinkwasser-Installation ist begrenzt. Darüber hinaus stehen lokale Kontaminationen im Gegensatz zu systemischen Kontaminationen in der Regel in engem Zusammenhang mit der individuellen Nutzung der beprobten Entnahmestelle.

\section{Geltungsbereich}

Diese Empfehlung gilt für systemische Untersuchungen von Trinkwasser-Installationen gemäß TrinkwV. Untersuchungen zur Feststellung von lokalen Kontaminationen sowie weitergehende Untersuchungen nach DVGW-Arbeitsblatt W 551 sind nicht Gegenstand dieser Empfehlung. Es wird jedoch empfohlen, für diese Untersuchungen in Bezug auf Transport/Lagerung der Proben (siehe Pkt. 5.3), Untersuchungsgang (siehe Pkt. 6) und Angabe der Ergebnisse (siehe Pkt. 7) analog zu verfahren.

Bei weitergehenden Untersuchungen gemäß DVGW W 551 wird empfohlen, auch die Probennahme gemäß dieser Empfehlung durchzuführen (siehe Pkt. 5). Bei infektionshygienischer Veranlassung, z. B. bei reaktiver Untersuchung zur Feststellung der Infektionsquelle nach Auftreten einer Legionelleninfektion oder nach einem Legionellenausbruch, kann auch eine Untersuchung zur Feststellung der Trinkwasserqualität an Entnahmestellen „so wie das Wasser verwendet wird“ notwendig sein. In diesem Fall ist eine Beprobung gemäß DIN EN ISO 19458, Tabelle 1, Zweck c) durchzuführen. Mit dieser Probennahmetechnik können lokale Kontaminationen an der untersuchten Entnahmearmatur festgestellt werden.

Die Untersuchung von Badebeckenwasser hat gemäß der DIN 19643 [5] zu erfolgen. Für die Laboruntersuchung kann diese Empfehlung sinngemäß angewendet werden (siehe Pkt. 5.3, Pkt. 6 und Pkt. 7). Um die geforderte Nachweisgrenze für die Bewertung des Parameters Legionellen zu gewährleisten, sind für Untersuchungen gemäß DIN 19643 insgesamt $100 \mathrm{ml} \mathrm{zu}$ filtrieren.

\section{Festlegung der Probennahmestellen}

Die Probennahmestellen, die für die Durchführung einer systemischen Untersuchung gemäß $\$ 14 \mathrm{~b}$ TrinkwV notwendig sind, beschreibt das DVGW-Arbeitsblatt W 551 (Abschnitt 9.1 orientierende Untersuchung). Nach $\$ 14$ b Absatz 3 TrinkwV müssen Proben für Untersuchungen auf den Parameter Legionella spec. an mehreren repräsentativen Probennahmestellen nach den a.a.R.d.T. entnommen werden. Von der Einhaltung dieser Regeln ist bei einer Probennahme nach DIN EN ISO 19458, Tabelle 1, Zweck b) auszugehen.

Bei der Probennahme nach DIN EN ISO 19458, Zweck b) wird der Einfluss der Entnahmearmatur so gering wie möglich gehalten. Die Probe soll die hygienischen Verhältnisse im Verteilungssystem des Gebäudes widerspiegeln.

In jeder Trinkwasser-Installation sind im Rahmen der systemischen Untersuchung (entspricht einer orientierenden Untersuchung) am Abgang der Leitung für Trinkwasser (warm) vom Trinkwassererwärmer sowie am Wiedereintritt in den Trinkwassererwärmer (Zirkulationsleitung) Proben zu entnehmen. Zusätzlich sind Proben in der Peripherie zu entnehmen. Die Entnahmestellen für die Proben in der Peripherie sind so zu wählen, dass jeder Steigstrang ${ }^{5}$ erfasst wird. Dies bedeutet nicht zwingend, dass Proben aus allen Steigsträngen $\mathrm{zu}$ entnehmen sind. Voraussetzung für die Auswahl ist, dass die beprobten Steigstränge eine Aussage über das Gesamtsystem, d.h. auch über die nicht beprobten Steigstränge, zulassen (z.B. weil sie ähnlich gebaut sind, gleichartige Gebäudebereiche versorgen und gleich genutzt werden oder möglichst hydraulisch ungünstig liegen $)^{6}$. Bei der Beprobung einer Auswahl von Steigsträngen ist die Repräsentativität dieser Probennahmestellen zu begründen.

Bei Trinkwasser-Installationen mit vielen Steigsträngen sind primär die Bereiche $\mathrm{zu}$ berücksichtigen, in denen es zur Vernebelung von Trinkwasser (z. B. beim Duschen) kommen kann.

Alternativ können auch alle Steigstränge beprobt werden. Der UsI der Trinkwasser-Installation entscheidet, ob er eine qualifizierte Person mit der Festlegung der zu untersuchenden Steigstränge beauftragt oder ob er alle Steigstränge beproben lässt. Insbesondere bei nur wenigen Steigsträngen kann letzteres einfacher sein.

Die Festlegung der Probennahmestellen liegt in der Verantwortung des UsI und ist durch hygienisch-technisch kompetentes Personal mit nachgewiesener Qualifikation zu treffen. Hinsichtlich der Anforderungen für eine ausreichende Qualifikation wird auf die Empfehlung des Umweltbundesamtes zur Durchführung einer Gefährdungsanalyse verwie$\operatorname{sen}^{7}$.

\footnotetext{
${ }^{5}$ Bei Trinkwasser-Installationen mit wenigen Steigsträngen und komplexen horizontalen Verteilungen sind auch endständige Entnahmestellen am Ende der horizontalen Verteilungsleitungen zu berücksichtigen.

6 Es wird empfohlen, dass mindestens alle Steigstränge mit einer Rücklauftemperatur $<55^{\circ} \mathrm{C}$ in die Beprobung einbezogen werden. In diesen Fällen kann davon ausgegangen werden, dass hydraulisch ungünstige Verhältnisse oder andere technische Mängel vorliegen.

7 Empfehlung des Umweltbundesamtes nach Anhörung der Trinkwasserkommission vom 14.Dezember 2012: Empfehlungen für die Durchführung einer Gefährdungsanalyse gemäß Trinkwasserverordnung. Maßnahmen bei Überschreitung des technischen Maßnahmenwertes für Legionellen.
} 
Die TrinkwV ${ }^{8}$ schreibt für eine systemische Untersuchung eine Probennahme gemäß DIN EN ISO 19458, Tabelle 1, Zweck b) vor. Eine Desinfektion der Probennahmearmatur ist erforderlich. Bei der Auswahl von Probennahmearmaturen sind daher abflammbare Ausführungen zu bevorzugen (siehe DIN EN ISO 19458 und VDI/DVGW 6023 Abschnitt 6.1). Unter der Probennahmearmatur muss genügend Abstand sein, damit Probennahmegefäße ohne Kontakt mit der Probennahmearmatur unter den Auslauf gehalten werden können. Es ist auf einfache Zugänglichkeit, auf Sauberkeit und geeignete Dokumentation der Probennahmestellen zu achten. Für die ordnungsgemäße Installation einer ausreichenden Anzahl von geeigneten Probennahmestellen ist der UsI in der Pflicht, dies ergibt sich nach $\$ 14$ b Absatz 3 Satz 4 TrinkwV und aus dem technischen Regelwerk (z. B. VDI/DVGW 6023 und DVGW-Arbeitsblatt W 551).

Die Entnahmestellen in den peripheren Teilen der Trinkwasser-Installation sollen in Bereichen mit Vernebelung liegen. Da es sich um eine systemische Untersuchung handelt, sollen Entnahmearmaturen an nahe gelegenen Waschbecken genutzt werden, eine Probennahme direkt an Duschköpfen ist für diesen Zweck zu vermeiden. Muss aufgrund der baulichen Situation die Probennahme am Duschkopf erfolgen, ist dies keine normenkonforme Probennahme und entspricht nicht der Probennahme nach Zweck b) und damit nicht den Anforderungen für eine systemische Untersuchung nach TrinkwV. Dieses Vorgehen ist zwingend im Probennahmeprotokoll $\mathrm{zu}$ vermerken und bei der Auswertung zu berücksichtigen. Periphere Proben sind an den Stellen mit der längsten Fließstrecke vom Trinkwassererwärmer zu entnehmen. Nicht genutzte Wohnungen oder nicht genutzte Entnahmestellen sind für die systemische

\footnotetext{
8 Hinweis: Neben den die Legionellen betreffenden Untersuchungspflichten nach TrinkwV können sich auch Pflichten aus anderen Rechtsbereichen ergeben z.B.:. aus Hygienebestimmungen in Risikobereichen (z.B. Krankenhaushygieneverordnungen der Bundesländer); aus der allgemeinen Verkehrssicherungspflicht (§823 BGB).
}

Untersuchung und deren Bewertung nicht repräsentativ.

Darüber hinaus kann das Gesundheitsamt gemäß $\$ 19$ Absatz 7 und $\$ 20$ TrinkwV anordnen, die Proben „an bestimmten Probennahmestellen nach bestimmten technischen Vorgaben zur Durchführung [...]“ zu entnehmen oder entnehmen zu lassen.

\section{Probennahme}

Nach $\$ 14$ b Absatz 2 dürfen Probennahme und Untersuchung nur durch für Trinkwasseruntersuchungen akkreditierte und nach $\$ 15$ Absatz 4 TrinkwV zugelassene Untersuchungsstellen erfolgen. Diese Untersuchungsstellen werden auf Listen der zuständigen obersten Landesbehörden bekannt gemacht. Externe Probennehmer müssen in das Qualitätsmanagementsystem des Laboratoriums eingebunden sein (siehe DIN EN ISO/IEC 17025 [6] und Erläuterungen dazu in der entsprechenden DAkkS-Regel 71 SD 4011 [7]). Eine Zertifizierung des Probennehmers allein genügt nicht den Anforderungen der TrinkwV. Die Durchführung der Probennahme und der Probentransport gehören zum Untersuchungsauftrag. Die Verantwortung für die Durchführung von Probennahme und Probentransport liegt bei der Laborleitung. Dies gilt auch im Hinblick auf die Unabhängigkeit und Unparteilichkeit der Probennehmer. Darüber hinaus ist durch den Probennehmer sicherzustellen, dass die Probennahme an geeigneten Probennahmestellen durchgeführt wird.

Unter bestimmten Bedingungen ist es erforderlich, auch die Trinkwasser-Installation für Trinkwasser (kalt) zu untersuchen, z. B. bei Feststellung einer Wassertemperatur $\geq 25^{\circ} \mathrm{C}$ nach Spülen der Entnahmestellen für $30 \mathrm{~s}$ im Trinkwasser (kalt) (siehe DIN 1988-200 [8] und DVGW-Information Wasser Nr. 90). Die Trinkwasser-Installation für Trinkwasser (warm) und die Trinkwasser-Installation für Trinkwasser (kalt) sind getrennt voneinander zu beproben.

Die Beprobung von Mischwasser ist zu vermeiden. Bei Einhebel-Mischbatterien ist dies nicht immer zu gewährleisten. Hier sind die Eckventile der nicht $\mathrm{zu}$ untersuchenden Zuleitungen vor der
Probennahme zu schließen. Falls nur Mischwasser für eine Probennahme zur Verfügung steht oder andere technische Probleme auftreten, so dass die Probennahme nicht den vorgenannten Punkten bzw. der DIN EN ISO 19458 entspricht, ist das auf dem Probennahmeprotokoll zu vermerken.

Die Probennahme erfolgt bei bestimmungsgemäßem Betrieb der TrinkwasserInstallation. Eine temporäre Erhöhung der Warmwasserspeichertemperatur, Spülungen oder eine Desinfektion der Trinkwasser-Installation vor der Probennahme widersprechen vorsätzlich dem Schutzzweck der Untersuchung nach TrinkwV.

Die Proben an allen geforderten Probennahmestellen sind am gleichen Kalendertag zu entnehmen. Wenn dies nicht möglich ist, müssen an den anderen Tagen, an denen weitere Proben genommen werden, zumindest die Proben aus den zentralen Teilen der Trinkwasser-Installation am Abgang vom Trinkwassererwärmer und am Wiedereintritt der Zirkulation in den Trinkwassererwärmer erneut entnommen und untersucht werden.

\subsection{Schritte der Probenahme - Regelung gilt für Trinkwasser (warm) und Trinkwasser (kalt)}

1. Vorbereitung der Entnahmestelle:

- Entfernung von Strahlreglern und anderen Vorrichtungen

- Desinfektion der Auslaufstelle der Entnahmearmatur, vorzugsweise durch Abflammen

2. Spülung der Entnahmearmatur:

- Öffnen der Entnahmearmatur

- Ablauf von 11 Trinkwasser in einen Messbecher

3. Befüllen des Probenbehälters:

- Abfüllen des Trinkwassers direkt anschließend (ohne Schließen und erneutes Wiederöffnen der Entnahmearmatur) in ein steriles Probennahmegefäß

- Verschließen des Gefäßes

- Verwerfen des Wassers aus dem Messbecher

4. Messung der Temperatur bei der Probennahme:

- Abfüllen von weiteren ca. $250 \mathrm{ml}$ Trinkwasser in einen Messbecher direkt anschließend 
- Unverzügliches Messen der Wassertemperatur

- Dokumentation als „Probennahmetemperatur"

5. Messung der Wassertemperatur bei Temperaturkonstanz:

- Ablaufen lassen des Trinkwassers aus der Entnahmearmatur bis zur Temperaturkonstanz in einen Messbecher

- Messen der Wassertemperatur im Messbecher

- Dokumentation als „Konstante Temperatur“

\subsection{Dokumentation}

Die Probennahme ist zu dokumentieren und in den Prüfbericht aufzunehmen. Folgende Angaben sollen zusätzlich zu den nach DIN EN ISO/IEC 17025 (siehe Abschnitt 7.3.3) geforderten enthalten sein:

- Name und Art des Gebäudes (z. B. Krankenhaus, Sporteinrichtung, Pflegeeinrichtung, Wohngebäude, vermietete Betriebsstätte)

- Bezeichnung des Gebäudeteils (z. B. Bauabschnitt, Stockwerk/Etage, Funktionsbereich, Raum)

- Lokale Lage der Entnahmestelle (z.B. Strang, Verteiler, Waschtisch, Spüle, Wanne, Dusche)

- Art der Entnahmestelle (z. B. Entnahmearmatur, Kugelhähne, EinhebelMischarmatur, Zweigriff-Mischarmatur, Armatur mit Verbrühungsschutz)

- Angaben zum Trinkwasser (z. B. erwärmtes Trinkwasser, kaltes Trinkwasser, Mischwasser)

- Ggf. betriebstechnische Besonderheiten während der Probennahme, wie z. B. der Ausfall oder die Zeitsteuerung der Zirkulationspumpe oder Wassererwärmung

\subsection{Transport und Lagerung}

Zum Transport und zur Lagerung der Proben enthalten sowohl die DIN EN ISO 19458 als auch die ISO 11731 Angaben. Danach sind die Proben so schnell wie möglich zur Untersuchungsstelle zu transportieren, vorzugsweise innerhalb eines Tages. Sie sind spätestens $48 \mathrm{~h}$ nach
Probennahme in der Untersuchungsstelle anzusetzen.

Die Proben sind während des Transportes lichtgeschützt und gekühlt bei (5 $\pm 3)^{\circ} \mathrm{C}$ aufzubewahren. Wird die Probe am selben Arbeitstag untersucht, können die Proben lichtgeschützt und bei Umgebungstemperatur transportiert werden.

Wasserproben (warm, $>60^{\circ} \mathrm{C}$ ) sollten direkt nach der Probennahme gekühlt werden. Der Transport von Wasserproben (kalt) und von Wasserproben (warm) muss in separaten Transportbehältnissen erfolgen.

Weitere Hinweise zur Probennahme finden sich in der DVGW twin Nr. 6 [9], in der DVGW-Wasser-Information Nr. 74 [10] sowie in der DVGW-Wasser-Information Nr. 90 [11].

\section{Untersuchungsgang}

Grundlage für die Durchführung der Untersuchungen sind die normativen Vorgaben der ISO 11731. Die in dieser Norm enthaltene Entscheidungsmatrix beschreibt verschiedene Untersuchungsgänge in Abhängigkeit von der Probenart und der zu erwartenden Begleitflora.

Für Trinkwässer kann von einer geringen Begleitflora ausgegangen werden, daher ist auf die in Anhang J Tabelle J.2 unter Matrix A genannten Verfahren zurückzugreifen. Bei der Erarbeitung dieser Empfehlung standen folgende Ziele im Vordergrund:

a) Dem Anspruch zur Durchführung systemischer Überwachungen von Trinkwasser-Installationssystemen soll auch weiterhin vollumfänglich entsprochen werden.

b) Es ist ein praktikables Untersuchungsverfahren zu beschreiben, das der Norm entspricht, aber den Untersuchungsaufwand so weit wie möglich begrenzt und damit auch keine übermäßige Erhöhung der Untersuchungskosten nach sich zieht.

c) Das Verfahren soll eine dem bisherigen Verfahren vergleichbar hohe Sicherheit bieten.

Unter Beachtung dieser Kriterien und auf Grundlage von Vergleichsuntersuchungen ist die Untersuchung folgendermaßen durchzuführen:
Anzuwendender Untersuchungsgang.

1. Direktes Ausplattieren (=Direktansatz) von $2 \times 0,5 \mathrm{ml}$ Wasserprobe auf $\mathrm{BCYE}+\mathrm{AB}$ Agar ohne Vorbehandlung; der Untersuchungsgang entspricht der Entscheidungsmatrix nach ISO 11731 Anhang J, Bild J.1, Verfahren 1 , Medium $\mathrm{BCYE}+\mathrm{AB}^{9}$.

2. Zusätzlich Membranfiltration der Wasserprobe mit Säurebehandlung und anschließendem Auflegen des Filters auf BCYE+AB Agar oder GVPC Agar; der Untersuchungsgang entspricht der Entscheidungsmatrix nach ISO 11731, Anhang J, Bild J.1, Verfahren 7, Medium BCYE+AB oder GVPC $^{10}$.

Für die Membranfiltration ist ein Volumen zwischen $50 \mathrm{ml}$ und $80 \mathrm{ml}$ einzusetzen, um die Anforderungen der ISO 8199 [12] hinsichtlich der oberen Arbeitsbereichsgrenze umzusetzen und den Messbereich des technischen Maßnahmenwertes (100 KBE/100 ml) sicher abzudecken.

\section{Angabe der Ergebnisse}

Gemäß der ISO 8199 ist die vertretbare Höchstanzahl an Zielkolonien auf einer Platte von dem jeweiligen Verfahren, der Koloniegröße, der Art der Kolonien und dem Vorhandensein von Nicht-Zielkolonien abhängig und kann auf den Ergebnissen von Verifizierungsversuchen beruhen. Um ein quantitatives Ergebnis anzugeben, sollten nur Platten ausgewertet werden, die als Richtwert einen maximalen Zählwert (Ziel- und Nicht-Zielkolonien) von 300 Kolonien beim Direktansatz und maximal 80 Kolonien bei der Membranfiltration aufweisen (ISO 8199).

Begleitorganismen können das Zählergebnis verfälschen oder das Wachstum

\footnotetext{
9 In ISO11731 wird zusätzlich das direkte Ausplattieren auf BCYE Agar ohne Vorbehandlung als erforderlicher Ansatz beschrieben. Vergleichsuntersuchungen in Trinkwasseruntersuchungsstellen in Deutschland haben jedoch gezeigt, dass dieses Verfahren aufgrund der geringen Selektivität des Mediums häufig zu keinen befriedigenden Ergebnissen führt. Die Platten weisen eine hohe Begleitflora auf, so dass eine Quantifizierung nicht immer möglich ist. Dieser Ansatz entfällt.

${ }_{10}$ Die Membranfiltration dient zur Senkung der Nachweisgrenze.
} 
Tab. 1 Beispiele für die Berechnung der Ergebnisse. Für die Berechnung der Ergebnisse aus den Direktansätzen dürfen nur die Ergebnisse

identischer Nährmedien berücksichtigt werden

Fall a: beide Platten des Direktansatzes sind auswertbar (unabhängig von der Auswertung der Membranfiltration)

Fall b: eine Platte des Direktansatzes ist auswertbar, eine Platte des Direktansatzes ist nicht auswertbar (unabhängig von der Auswertung der Membranfiltration)

Fall c: alle Platten des Direktansatzes sind nicht auswertbar, Platte der Membranfiltration auswertbar

Fall d: Direktansätze auswertbar, Membranfiltration ist nicht auswertbar

Fall e: Ergebnis aus dem Direktansatz $=0$, Ergebnis aus der Membranfiltration $=0$

Fall f: Direktansatz und Membranfiltration enthalten mehr Kolonien als für die Angabe eines quantitativen Ergebnisses zulässig wäre (mehr als 300 Kolonien beim Direktansatz bzw. mehr als 80 Kolonien bei der Membranfiltration)

Fall g: Membranfiltration enthält mehr verdächtige Kolonien als für die Angabe eines quantitativen Ergebnisses zulässig wäre (mehr als 80 Kolonien; Direktansatz $=0$ oder nicht auswertbar)

Fall h: Direktansatz und Membranfiltration enthalten zu viele Kolonien und sind nicht quantitativ auswertbar; durch Bestätigung von einzelnen Kolonien werden nur Begleitkeime bestätigt (keine Legionellen)

Fall i: Membranfiltration und Direktansätze enthalten zu viele Kolonien und sind daher überhaupt nicht auswertbar (Rasen)

aBeispiel für die Berechnung der Ergebnisse aus dem Direktansatz: Eine Platte enthält 3 Kolonien, die zweite Platte aus dem Direktansatz enthält eine Kolonie. Ergebnis: 1 ml Probe enthalten 4 Kolonien; es wurden $400 \mathrm{KBE} / 100 \mathrm{ml}$ gefunden

bBei der Ergebnisangabe ist das tatsächlich eingesetzte Volumen für die Membranfiltration zu berücksichtigen und entsprechend anzugeben
Die Koloniezahlen der beiden Direktansätze von je 0,5 ml Probe werden addiert, die Summe mit 100 multipliziert und als KBE/100 ml angegeben ${ }^{\text {a }}$

Die Koloniezahlen des auswertbaren Direktansatzes werden ausgezählt, verdoppelt, mit 100 multipliziert und als KBE/100 ml angegeben

Ergebnisangabe aus der Membranfiltration nach entsprechender Hochrechnung

Ergebnisangabe erfolgt aus den Direktansätzen nach entsprechender Hochrechnung

Wenn weder auf den Platten aus dem Direktansatz noch auf der Platte der Membranfiltration Legionellen nachgewiesen werden, ist als Ergebnis "nicht nachgewiesen (n. n.) in $50 \mathrm{ml}^{\prime \prime}$ anzugeben ${ }^{\mathrm{b}}$. Gemäß ISO 8199 sind auch Angaben wie" $<2 \mathrm{KBE} / 100 \mathrm{ml}$ " zulässig

Als Ergebnis ist anzugeben "> $160 \mathrm{KBE} / 100 \mathrm{ml}^{\mathrm{b}}$ Legionellen"

Als Ergebnis ist anzugeben „nicht auswertbar". Eine Nachprobe ist erforderlich

Als Ergebnis ist "nicht auswertbar" anzugeben. Eine Nachprobe ist erforderlich
Als Ergebnis ist anzugeben "> $60.000 \mathrm{KBE} / 100 \mathrm{ml}$ Legionellen" von Legionellen vollständig verhindern. Ergebnisse, die unter Einbeziehung von Platten, die sehr viele Begleitorganismen aufweisen, erzielt wurden, sind daher mit einer Unsicherheit behaftet. Wenn möglich ist auf die Einbeziehung von Platten mit starker Kontamination durch Begleitorganismen (mehr Begleitorganismen als Legionellen) zur Berechnung des Ergebnisses zu verzichten. Wenn nur Platten zur Auswertung vorliegen, die starke Kontamination durch Begleitorganismen aufweisen, ist die Untersuchung zu wiederholen. Ist eine Wiederholung unmöglich oder ist auch bei wiederholter Durchführung der Probennahme keine Platte zu erhalten, die weitgehend frei von Begleitorganismen ist, dann muss dieser Umstand im Prüfbericht angegeben werden.

Aus Gründen des vorbeugenden Gesundheitsschutzes ist das höhere der Ergebnisse aus den Direktansätzen und der Membranfiltration, bezogen auf $100 \mathrm{ml}$ Untersuchungsvolumen, anzugeben.
Auf dem Prüfbericht ist auszuweisen, aus welchem Ansatz das Endergebnis bestimmt wurde. Dies kann z. B. durch das normgerechte Zitieren des verwendeten Verfahrens erfolgen (siehe Pkt. 6 anzuwendender Untersuchungsgang). Beispiele für die Berechnung der Ergebnisse werden in $\bullet$ Tab. 1 genannt.

\section{Bewertung der Ergebnisse}

Bei der Bewertung der Untersuchungsergebnisse sind die folgenden rechtlichen und normativen Regularien zu berücksichtigen:

- TrinkwV: technischer Maßnahmenwert nach Anlage 3 Teil II von 100 $\mathrm{KBE} / 100 \mathrm{ml}$

- DVGW W 551: Bewertung der Untersuchungsergebnisse aus der systemischen $=$ orientierenden Untersuchung nach Tabelle 1a

- DVGW W 551: Bewertung der Untersuchungsergebnisse aus der weiterge- henden Untersuchung nach Tabelle $1 b$

- Empfehlung des UBA zur periodischen Untersuchung auf Legionellen in zentralen Trinkwassererwärmungsanlagen $^{11}$ : Bewertung unter Berücksichtigung spezifischer Anforderungen in medizinischen Einrichtungen.

Darüber hinaus besteht eine Anzeigepflicht für Trinkwasseruntersuchungsstellen ( $\$ 15 \mathrm{a}$ TrinkwV) bei Überschreitung des Technischen Maßnahmenwertes von $100 \mathrm{KBE} / 100 \mathrm{ml}$.

Die Ergebnisse aus Untersuchungen nach Probennahme gemäß DIN EN

11 Empfehlung des Umweltbundesamtes nach Anhörung der Trinkwasserkommission des Bundesministeriums für Gesundheit: Periodische Untersuchung auf Legionellen in zentralen Erwärmungsanlagen der Hausinstallation nach $\S 3$ Nr.2 Buchstabec TrinkwV 2001, aus denen Wasser für die Öffentlichkeit bereitgestellt wird (Bundesgesundheitsbl - Gesundheitsforsch Gesundheitsschutz 2006 S.697). 
ISO 19458, Tabelle 1, Zweck c) können nicht zur Umsetzung der Anforderungen gemäß $\$ 14$ b TrinkwV oder der Anforderungen gemäß DVGW-Arbeitsblatt W 551 verwendet oder bewertet werden. Diese Untersuchungen gehen über die Untersuchung zur Feststellung einer systemischen Kontamination hinaus, sie können allerdings über $\$ 19$ Absatz 7 oder $\$ 20$ der TrinkwV durch die Gesundheitsämter veranlasst werden bzw. im Rahmen von weitergehenden Untersuchungen erforderlich sein. Eine Bewertung der Untersuchungsergebnisse erfolgt im Einzelfall unter Berücksichtigung der konkreten Situation und der Bedingungen bei der Probennahme und liegt im Ermessen der Gesundheitsämter.

\section{Literatur}

1. DIN EN ISO 19458: 2012-06: Wasserbeschaffenheit - Probenahme für mikrobiologische Untersuchungen

2. ISO 11731: 2017-05: Wasserbeschaffenheit - Zählung von Legionellen

3. DIN EN ISO 11731:2018-03: Wasserbeschaffenheit - Zählung von Legionellen (ISO 11731:2017)

4. DVGW-Arbeitsblatt W 551: Trinkwassererwärmungs- und Trinkwasserleitungsanlagen - Technische Maßnahmen zur Verminderung des Legionellenwachstums - Planung, Errichtung, Betrieb und Sanierung von Trinkwasser-Installationen

5. DIN 19643:2006-12: Aufbereitung von Schwimmund Badebeckenwasser, Beuth-Verlag, Berlin

6. DIN EN ISO/IEC 17025: 2018-03: Allgemeine Anforderungen an die Kompetenz von Prüf- und Kalibrierlaboratorien (ISO/IEC 17025: 2017)

7. DAkkS-Regel 71 SD 4011 , Revision 1.4 vom 19.01.2017: Anforderungen bei der Akkreditierung von Untersuchungsstellen für Trinkwasser

8. DIN 1988-200:2012-05: Technische Regeln für Trinkwasser-Installationen - Teil 200: Installation Typ A (geschlossenes System) - Planung, Bauteile, Apparate, Werkstoffe; Technische Regel des DVGW

9. DVGW twin Nr. 6: Durchführung der Probennahme zur Untersuchung des Trinkwassers auf Legionellen (ergänzende systemische Untersuchung von Trinkwasser-Installationen), DVGW, Bonn

10. DVGW-Wasserinformation Nr. 74 (01/2012): Hinweise zur Durchführung von Probennahmen aus der Trinkwasser-Installation für die Untersuchung auf Legionellen, DVGW, Bonn

11. DVGW-Wasserinformation Nr. 90 (03/2017): Informationen und Erläuterungen zu Anforderungen des DVGW-Arbeitsblattes W 551, DVGW, Bonn

12. ISO 8199:2018: Water quality-General requirements and guidance for microbiological examinations by culture 